\title{
Differential positivity on compact sets
}

\author{
F. Forni
}

\begin{abstract}
The paper studies differentially positive systems, that is, systems whose linearization along an arbitrary trajectory is positive. Extending the results in [7], we illustrate the use of differential positivity on compact forward invariant sets for the characterization of bistable and periodic behaviors. Geometric conditions for differential positivity are provided. The introduction of compact sets simplifies the use of differential positivity in applications.
\end{abstract}

\section{INTRODUCTION}

A linear system is positive if some cone $\mathcal{K}$ on the system state space is invariant for the dynamics [3]. Positivity strongly restricts the behavior of a linear system. Under mild conditions, the ray $\lambda v \in \mathcal{K}$ given by the Perron-Frobenius eigenvector $v \in \mathcal{K}$ is an attractor for the system dynamics, [2], [3]. This fundamental property is exploited in a number of applications [5], [12], [15], [14]

Differential positivity extends linear positivity to the nonlinear setting. A nonlinear system is differentially positive if its linearization along trajectories makes a cone (field) invariant [7]. Differential positive systems are a large class of systems encompassing monotone systems [1], [9], [18]. Under mild conditions, the trajectories of a differentially positive system converge to a one dimensional attractor, a relevant property for the study of bistable and periodic behaviors. In comparison to linear positivity, this attractor is not a ray, but a curve, possibly closed in the presence of attractive limit cycles.

In this paper we make differential positivity readily available for the analysis of nonlinear systems by deriving a number of geometric tools. The problem of establishing the differential positivity of a system is encoded into a set of pointwise geometric conditions to test. Differential positivity is then used to derive novel methods for the analysis of simple attractors of nonlinear systems, typically capturing bistable and periodic behaviors. Compactness simplifies the use of differential positivity in applications. In particular, the restriction to compact and forward invariant sets makes the geometric conditions for differential positivity much simpler to verify in practice.

Section II introduces the notion of cone fields and characterizes their representation as a set of inequalities. The two

F. Forni is with the University of Cambridge, Department of Engineering, Trumpington Street, Cambridge CB2 1PZ, and with the Department of Electrical Engineering and Computer Science, University of Liège, 4000 Liège, Belgium, ff286@cam.ac.uk. The research was supported by the Fund for Scientific Research FNRS and by the Engineering and Physical Sciences Research Council under Grant EP/G066477/1. The paper presents research results of the Belgian Network DYSCO (Dynamical Systems, Control, and Optimization), funded by the Interuniversity Attraction Poles Programme, initiated by the Belgian State, Science Policy Office. The scientific responsibility rests with its authors. large families of polyhedral and quadratic cone fields are illustrated. Section III recalls the basic notions of differential positivity. Section IV provides a set of geometric conditions for testing the differential positivity of a system. The use of the geometric conditions is illustrated on a cooperative system and on a nonlinear pendulum. The role of differential positivity for the analysis of the asymptotic nonlinear behavior is discussed in Section $\mathrm{V}$ and illustrated on the Kuramoto model in Section VI. Conclusions follow.

\section{CONE FIELDS}

We recall some basic geometric notions on Riemannian manifolds which will be useful to the discussion on differential positivity. Let $\mathcal{X}$ be a smooth $n$-dimensional manifold endowed with a Riemannian metric $\langle\cdot, \cdot\rangle_{x}: T_{x} \mathcal{X} \times T_{x} \mathcal{X} \rightarrow \mathbb{R}$ where $T_{x} \mathcal{X}$ denotes the tangent space at $x \in \mathcal{X}$. We will use $T \mathcal{X}$ to denote the tangent bundle of $\mathcal{X}$, and $|\delta x|_{x}$ to denote $\sqrt{\langle\delta x, \delta x\rangle_{x}}$ for all $\delta x \in T_{x} \mathcal{X}$. Furthermore, given any function $\psi: \mathcal{X} \rightarrow \mathcal{Y}$ between manifolds, $\partial \psi(x): T_{x} \mathcal{X} \rightarrow$ $T_{\psi(x)} \mathcal{Y}$ will denote the differential of $\psi$ at $x \in \mathcal{X}$. Given any set $\mathcal{S} \subseteq T_{x} \mathcal{X}$ we will write $\partial \psi(x) \mathcal{S}:=\{\psi(x) \delta x \mid \delta x \in \mathcal{S}\}$.

To extend linear positivity into the differential setting, we will exploit the notion of conal manifold, that is, a manifold $\mathcal{X}$ endowed with a cone field

$$
\mathcal{K}_{\varepsilon}(x) \subseteq T_{x} \mathcal{X} \quad \forall x \in \mathcal{X}
$$

where $0 \leq \varepsilon \ll 1$. At any $x, \mathcal{K}_{\varepsilon}(x)$ is just a cone in the vector space $T_{x} \mathcal{X}$. The role of the parameter $\varepsilon$ is clarified by the property $\mathcal{K}_{\varepsilon_{2}}(x) \backslash\{0\} \subset \mathcal{K}_{\varepsilon_{1}}(x) \backslash\{0\}$ if $\varepsilon_{1}<\varepsilon_{2}$, that we assume throughout the paper. We use $\mathcal{K}(x):=\mathcal{K}_{0}(x)$.

For each $\varepsilon$, each cone $\mathcal{K}_{\varepsilon}(x)$ is closed and solid, and satisfies (i) $\mathcal{K}_{\varepsilon}(x)+\mathcal{K}_{\varepsilon}(x) \subseteq \mathcal{K}_{\varepsilon}(x)$, (ii) $\alpha \mathcal{K}_{\varepsilon}(x) \subseteq \mathcal{K}_{\varepsilon}(x)$ for all $\alpha>0$, (iii) $\mathcal{K}_{\varepsilon}(x) \cap-\mathcal{K}_{\varepsilon}(x)=\{0\}$, which make $\mathcal{K}_{\varepsilon}(x)$ convex and pointed. To avoid pathological cases, we assume that for every $x_{1}, x_{2} \in \mathcal{X}$, there exists a linear invertible mapping $\Gamma\left(x_{1}, x_{2}\right): T_{x_{1}} \mathcal{X} \rightarrow T_{x_{2}} \mathcal{X}$ such that, for any $\varepsilon, \Gamma\left(x_{1}, x_{2}\right) \mathcal{K}_{\varepsilon}\left(x_{1}\right)=\mathcal{K}_{\varepsilon}\left(x_{2}\right)$. Cone fields satisfying this property are said regular.

To make use of a cone field in computations, the cone field $\mathcal{K}_{\varepsilon}(x)$ will be represented by

$$
\delta x \in \mathcal{K}_{\varepsilon}(x) \backslash\{0\} \Leftrightarrow K_{i}\left(x, \frac{\delta x}{|\delta x|_{x}}\right) \geq \varepsilon, \forall i \in \mathcal{I} \subseteq \mathbb{N}
$$

where each $K_{i}: T \mathcal{X} \rightarrow \mathbb{R}$ is a smooth function. For simplicity, in what follows we will use $K \geq \varepsilon$ to denote the component-wise inequality $K_{i} \geq \varepsilon$ for each $\forall i \in \mathcal{I}$. For the cone $\mathcal{K}(x)$, (2) is equivalent $K_{i}(x, \delta x) \geq 0$. The normalization $\frac{\delta x}{|\delta x|_{x}}$ in (2) maps every ray $\lambda \delta x \in \mathcal{K}(x), \lambda>$ 0 , into the point $\frac{\delta x}{|\delta x|_{x}}$ of the unit sphere $\left\{\left.v \in T_{x} \mathcal{X}|| v\right|_{x}=\right.$ 
$1\}$. The normalization makes the representation independent of the length of the tangent vectors $\delta x$. Examples of smoothly varying polyhedral and quadratic cone fields are provided at the end of the section. A simple illustration is in Figure 1.

A cone field carries naturally the useful notion of conal curve $\gamma: \mathbb{R} \rightarrow \mathcal{X}$, which is an integral curve of the cone field

$$
\dot{\gamma}(s) \in \mathcal{K}(\gamma(s)) \quad \forall s \in \mathbb{R} .
$$

We make the standing assumption that $|\dot{\gamma}(s)|_{\gamma(s)}=1$ for any $s \in \mathbb{R}$. From (2), a conal curve satisfies $K(\gamma(s), \dot{\gamma}(s)) \geq 0$.
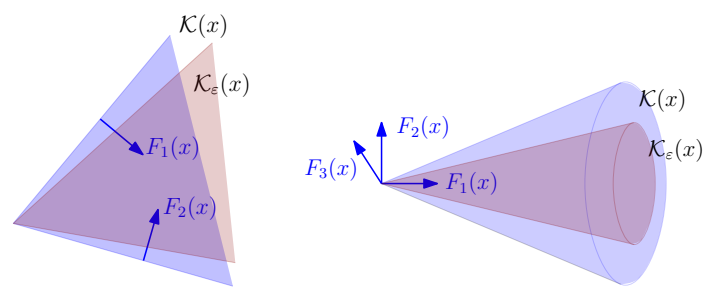

Fig. 1. Polyhedral and quadratic cones at $T_{x} \mathcal{X}$.

Example 1: polyhedral cone fields. Let $\mathcal{X}$ be a smooth manifold of dimension $n$ endowed with a Riemannian metric $\langle\cdot, \cdot\rangle_{x}$. For any $i \in \mathcal{I}:=\{1, \ldots, m\} \subseteq \mathbb{N}, m \geq n$, define

$$
K_{i}(x, \delta x):=\left\langle F_{i}(x), \delta x\right\rangle_{x}
$$

where $F_{i}(x) \in T_{x} \mathcal{X} \backslash\{0\}$. We assume that: $\mathbf{A 1}$ for every pair of points $x_{1}, x_{2} \in \mathcal{X}$, there exists a linear invertible isometry $T\left(x_{1}, x_{2}\right): T_{x_{1}} \mathcal{X} \rightarrow T_{x_{2}} \mathcal{X}$ such that $F_{i}\left(x_{2}\right)=$ $T\left(x_{1}, x_{2}\right) F_{i}\left(x_{1}\right)$; $\mathbf{A 2}\left\{F_{1}, \ldots, F_{m}\right\}$ is a smooth full-rank distribution; $\mathbf{A} \mathbf{3}$ the set of constraints (2),(4) is feasible for some $\bar{\varepsilon}>0$. Then, for all $0 \leq \varepsilon<\bar{\varepsilon}$, the cone field $\mathcal{K}_{\varepsilon}(x)$ given by (2),(4) is solid, pointed, convex and regular.

Assumption $\mathbf{A 3}$ guarantees that, for $\varepsilon<\bar{\varepsilon}, \mathcal{K}_{\varepsilon}(x)$ is solid, pointed and convex by construction, since the distribution is full-rank. For regularity, consider any pair of points $x_{1}, x_{2} \in \mathcal{X}$, and take $\left\langle F_{i}\left(x_{1}\right), \frac{\delta x_{1}}{\left|\delta x_{1}\right| x_{1}}\right\rangle_{x_{1}}=$ $\varepsilon$ and $\delta x_{2}=T\left(x_{1}, x_{2}\right) \delta x_{1}$. Then, $\left\langle F_{i}\left(x_{2}\right), \delta x_{2}\right\rangle_{x_{2}}=$ $\left\langle T\left(x_{1}, x_{2}\right) F_{i}\left(x_{1}\right), T\left(x_{1}, x_{2}\right) \delta x_{1}\right\rangle_{x_{2}}=\left\langle F_{i}\left(x_{1}\right), \delta x_{1}\right\rangle_{x_{1}}=$ $\varepsilon\left|\delta x_{1}\right|_{x_{1}}$. Thus, $\left\langle F_{i}\left(x_{2}\right), \frac{\delta x_{2}}{\left|\delta x_{2}\right| x_{2}}\right\rangle_{x_{2}}=\varepsilon \frac{\left|\delta x_{1}\right| x_{1}}{\left|\delta x_{2}\right| x_{2}}=\varepsilon$ since $T\left(x_{1}, x_{2}\right)$ is an isometry.

Example 2: quadratic cone fields. Let $\mathcal{X}$ be a smooth manifold of dimension $n$ endowed with a Riemannian metric $\langle\cdot, \cdot\rangle_{x}$. Consider $m \geq n$ vector fields $F_{i}(x) \in T_{x} \mathcal{X} \backslash\{0\}$ and define

$$
\begin{aligned}
& K_{1}(x, \delta x):=\left\langle F_{1}(x), \delta x\right\rangle_{x} \\
& K_{2}(x, \delta x):=\left\langle F_{1}(x), \delta x\right\rangle_{x}^{2}-\sum_{i, j \in\{2, \ldots, n\}}\left\langle F_{i}(x), \delta x\right\rangle_{x}\left\langle F_{j}(x), \delta x\right\rangle_{x} .
\end{aligned}
$$

Assumptions A1-A3 with the additional condition A4 $\left\langle F_{1}(x), F_{i}(x)\right\rangle_{x}=0$ for any $i>1$, guarantee that the cone field (2), (5) is solid, pointed, convex and regular for $\varepsilon<\bar{\varepsilon}$.

Note that $K_{2} \geq 0$ characterizes a double cone which is refined to a pointed cone by $K_{1} \geq 0$. The cone fields $\mathcal{K}$ and $\mathcal{K}_{\varepsilon}$ are solid and convex by construction. Regularity follows from the observation that $\left\langle F_{i}\left(x_{2}\right), \delta x_{2}\right\rangle_{x_{2}}=\left\langle F_{i}\left(x_{1}\right), \delta x_{1}\right\rangle_{x_{1}}$ for each $x_{1}, x_{2} \in \mathcal{X}$ and each $\delta x_{2}=T\left(x_{1}, x_{2}\right) \delta x_{1}$.

\section{DifFERENTIAL POSITIVITY}

\section{A. Differential positivity in forward invariant regions}

A linear system $\dot{x}=A x, x \in \mathbb{R}^{n}$, is positive if there exists a cone $\mathcal{K} \subseteq \mathbb{R}^{n}$ which is forward invariant for the system dynamics, i.e. $e^{A t} \mathcal{K} \subseteq \mathcal{K}$ for $t \geq 0$, [3]. Differential positivity extends linear positivity to nonlinear dynamics

$$
\Sigma: \dot{x}=f(x) \quad x \in \mathcal{X},
$$

by requiring that a given cone field is forward invariant for the prolonged dynamics [4],

$$
\delta \Sigma:\left\{\begin{aligned}
\dot{x} & =f(x) \\
\dot{\delta x} & =\partial f(x) \delta x \quad(x, \delta x) \in T \mathcal{X} .
\end{aligned}\right.
$$

(7) represents the linearization of $\Sigma$ along its trajectories. For simplicity we assume $f \in C^{2}$ and forward completeness of $\Sigma$. We use $\psi\left(t, x_{0}\right): \mathbb{R} \times \mathcal{X} \rightarrow \mathcal{X}$ to denote the state reached at time $t$ by the trajectory of $\Sigma$ from the initial condition $x_{0}$. Indeed, $\psi\left(\cdot, x_{0}\right) \in \Sigma$. We also use $\psi_{t}(\cdot): \mathcal{X} \rightarrow$ $\mathcal{X}$, which maps any $x \in \mathcal{X}$ into $\psi_{t}(x):=\psi(t, x)$. Finally, for any $(x, \delta x) \in T \mathcal{X}$, note that $\left(\psi(\cdot, x), \partial_{x} \psi(\cdot, x) \delta x\right) \in \delta \Sigma$.

Revisiting the definitions in [7], consider any forward invariant region $\mathcal{C} \subseteq \mathcal{X}$. We say that $\Sigma$ is differentially positive in $\mathcal{C}$ with respect to the cone field $\mathcal{K}(x)$ if for all $x \in \mathcal{C}$ and $t \geq 0$,

$$
\partial \psi_{t}(x) \mathcal{K}(x) \subseteq \mathcal{K}\left(\psi_{t}(x)\right) .
$$

Differential positivity captures the invariance of the cone field along the linearized dynamics. Furthermore, we say that $\Sigma$ is (uniformly) strictly differentially positive if it is differentially positive and there exists $T>0$ and $\varepsilon>0$ such that, for all $x \in \mathcal{C}$ and $t \geq T$,

$$
\partial \psi_{t}(x) \mathcal{K}(x) \subseteq \mathcal{K}_{\varepsilon}\left(\psi_{t}(x)\right) .
$$

Strict differential positivity captures the contraction of the cone field $\mathcal{K}(x)$ along the linearized dynamics, as shown in Figure 2.

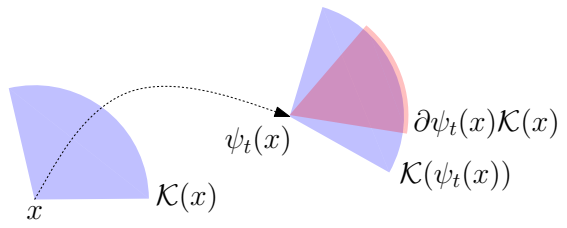

Fig. 2. Differential positivity: forward invariance of the cone field. Strict differential positivity: contraction of the rays of the cone field.

\section{B. Contraction of the Hilbert metric}

The contraction of the cone field along trajectories has a metric characterization based on the Hilbert metric, [3]. From [7, Section VI], for any given $x \in \mathcal{C}$, take any $\delta x, \delta y \in$ $\mathcal{K}(x) \backslash\{0\}$ and define $M_{\mathcal{K}(x)}(\delta x, \delta y):=\inf \{\lambda \geq 0 \mid \lambda \delta y-$ $\delta x \in \mathcal{K}(x)\}^{1}$ and $m_{\mathcal{K}(x)}(\delta x, \delta y):=\sup \{\lambda \geq 0 \mid \delta x-\lambda \delta y \in$ $\mathcal{K}(x)\}$. The Hilbert metric $d_{\mathcal{K}(x)}$ reads

$$
\begin{gathered}
d_{\mathcal{K}(x)}(\delta x, \delta y):=\log \left(\frac{M_{\mathcal{K}(x)}(\delta x, \delta y)}{m_{\mathcal{K}(x)}(\delta x, \delta y)}\right) \\
{ }^{1} M_{\mathcal{K}(x)}(\delta x, \delta y):=\infty \text { when }\left\{\lambda \in \mathbb{R}_{\geq 0} \mid \lambda \delta y-\delta x \in \mathcal{K}(x)\right\}=\emptyset .
\end{gathered}
$$


$d_{\mathcal{K}(x)}$ measures the distance between rays of the cone. It defines a metric in $\mathcal{K}(x) \cap\left\{\left.\delta x \in T_{x} \mathcal{X}|| \delta x\right|_{x}=1\right\}$. Furthermore, for any $\delta x, \delta y \in \mathcal{K}(x), d_{\mathcal{K}(x)}(\delta x, \delta y)=0$ if and only if $\delta x=\lambda \delta y$ with $\lambda \geq 0$, and $d_{\mathcal{K}(x)}(\alpha \delta x, \beta \delta y)=$ $d_{\mathcal{K}(x)}(\delta x, \delta y)$ for any $\alpha>0$ and $\beta>0$.

The contraction of the cone field along trajectories is captured by the exponential convergence of the Hilbert metric, as stated by the next lemma.

Lemma 1: [7, Theorem 2]. Let $\Sigma$ be a strictly differentially positive system with respect to the cone field $\mathcal{K}(x)$ in the forward invariant set $\mathcal{C} \subseteq \mathcal{X}$. Then, there exist $k \geq 1$ and $\lambda>0$ such that, for all $x \in \mathcal{C}, \delta x_{1}, \delta x_{2} \in \mathcal{K}(x)$, and $t \geq T$,

$$
d_{\mathcal{K}\left(\psi_{t}(x)\right)}\left(\partial \psi_{t}(x) \delta x_{1}, \partial \psi_{t}(x) \delta x_{2}\right) \leq k e^{-\lambda(t-T)} \Delta
$$

where $\left.\Delta:=\sup \left\{d_{\mathcal{K}(x)}\left(v_{1}, v_{2}\right) \mid v_{1}, v_{2} \in \mathcal{K}_{\varepsilon}(x)\right\}<\infty . \quad\right\lrcorner$

Lemma 1 and the following (mild) technical assumption are crucial for the theorems of Section V.

Assumption 1: $\left(\mathcal{K}(x) \cap\left\{\left.\delta x \in T_{x} \mathcal{X}|| \delta x\right|_{x}=1\right\}, d_{\mathcal{K}(x)}\right)$ is a complete metric space for all $x \in \mathcal{C}$.

The reader is referred to [3, Section 4], [10, Section 2.5], or [19] for examples of complete metric spaces on cones.

\section{GeOMETRIC CONDitions}

To provide geometric conditions for differential positivity we first reformulate the property using the representation (2). For instance, $\Sigma$ is differentially positive if for all $t \geq 0$ and all $(x, \delta x) \in T \mathcal{X}$,

$$
K(x, \delta x) \geq 0 \Rightarrow K\left(\psi_{t}(x), \partial \psi_{t}(x) \delta x\right) \geq 0 .
$$

In addition, $\Sigma$ is strictly differentially positive if there exists $T>0$ and $\varepsilon>0$ such that, for all $t \geq T$ and all $(x, \delta x) \in T \mathcal{X}$,

$$
K(x, \delta x) \geq 0 \Rightarrow K\left(\psi_{t}(x), \frac{\partial \psi_{t}(x) \delta x}{\left|\partial \psi_{t}(x) \delta x\right|_{\psi_{t}(x)}}\right) \geq \varepsilon
$$

(12) and (13) capture the invariance and the contraction of the cone field along trajectories, which leads to the following pointwise geometric conditions for differential positivity.

Theorem 1: $\Sigma$ is differentially positive in the forward invariant set $\mathcal{C} \subseteq \mathcal{X}$ with respect to $\mathcal{K}(x)$ if for any $(x, \delta x) \in$ $T \mathcal{X}$ such that $K(x, \delta x) \geq 0$ and $x \in \mathcal{C}$,

$$
K_{i}(x, \delta x)=0 \Rightarrow \partial K_{i}(x, \delta x)\left[\begin{array}{c}
f(x) \\
\partial f(x) \delta x
\end{array}\right] \geq 0 .
$$

Proof: Whenever $\left(\psi(\cdot, x), \partial_{x} \psi(\cdot, x) \delta x\right) \in \delta \Sigma$ reaches the boundary of the cone field at time $t$, we have $K\left(\psi(t, x), \partial_{x} \psi(t, x) \delta x\right) \geq 0$ and $K_{i}\left(\psi(t, x), \partial_{x} \psi(t, x) \delta x=\right.$ 0 , for some $i \in \mathcal{I}$. From (14), $\frac{d}{d t} K_{i}\left(\psi(t, x), \partial_{x} \psi(t, x) \delta x \geq\right.$ 0 , thus $K_{i}\left(\psi(t, x), \partial_{x} \psi(t, x) \delta x\right)$ either grows positive or remains at zero.

For strict differential positivity we need to take into account vectors on the unit sphere. For instance, for any $\delta x \in T_{x} \mathcal{X}$, consider $\vartheta:=\frac{\delta x}{|\delta x|_{x}}$. From (7),

$$
\dot{\vartheta}=(\partial f(x)-\lambda(x, \vartheta)) \vartheta
$$

where $\lambda(x, \vartheta)$ normalizes the action of the operator $\partial f(x)$ to guarantee that any trajectory $\vartheta(\cdot)$ of (15) from $|\vartheta(0)|_{x(0)}=1$ satisfies $|\vartheta(t)|_{x(t)}=1$ for all $t>0$. For example, given the representation $|\delta x|_{x}:=\left(\delta x^{T} G(x) \delta x\right)^{\frac{1}{2}}$, where $G(x)$ is the Riemannian tensor in local coordinates, $\lambda(x, \vartheta):=$ $\frac{1}{2} \vartheta^{T}\left(G(x) \partial f(x)+\partial f(x)^{T} G(x)+\sum_{i=1}^{n} \partial_{x_{i}} G(x) f(x)_{i}\right) \vartheta$. $\left(f(x)_{i}\right.$ is the $i$ th component of the vector $\left.f(x)\right)$.

Theorem 2: $\Sigma$ is strictly differentially positive in the forward invariant set $\mathcal{C} \subseteq \mathcal{X}$ with respect to $\mathcal{K}(x)$ if there exist $T>0$ and $\varepsilon>0$ such that, for any $(x, \vartheta) \in T \mathcal{X}$ that satisfies $K(x, \vartheta) \geq 0, x \in \mathcal{C}$ and $|\vartheta|_{x}=1$,

$$
\begin{aligned}
& 0 \leq K_{i}(x, \vartheta) \leq \varepsilon \Rightarrow \\
& \partial K_{i}(x, \vartheta)\left[\begin{array}{c}
f(x) \\
(\partial f(x)-\lambda(x, \vartheta)) \vartheta
\end{array}\right] \geq \frac{\varepsilon}{T} .
\end{aligned}
$$

Proof: Consider any trajectory $\left(\psi(\cdot, x), \partial_{x} \psi(\cdot, x) \delta x\right) \in$ $\delta \Sigma$. For simplicity, define $\vartheta(t):=\frac{\partial_{x} \psi(t, x) \delta x}{\left|\partial_{x} \psi(t, x) \delta x\right|_{\psi(t, x)}}$ and $x(t):=\psi(t, x)$. Differential positivity follows from the argument of Theorem 1. For strict differential positivity, suppose that for all $t \in[0, T], 0 \leq K_{i}(x(t), \vartheta(t))<\varepsilon$. Then, (16) guarantees that $K_{i}(x(T), \vartheta(T))=K_{i}(x(0), \vartheta(0))+$ $\int_{0}^{T} \frac{d}{d t} K_{i}(x(t), \vartheta(t)) d t \geq \int_{0}^{T} \frac{\varepsilon}{T} d t=\varepsilon$. A contradiction.

Because of the normalization, verifying (16) may be a daunting task in practice. However, those conditions become simpler when the forward invariant region $\mathcal{C}$ is compact.

Theorem 3: $\Sigma$ is strictly differentially positive in the compact and forward invariant set $\mathcal{C} \subseteq \mathcal{X}$ with respect to $\mathcal{K}(x)$ if for any $(x, \vartheta)$ such that $K(x, \vartheta) \geq 0, x \in \mathcal{C}$ and $|\vartheta|_{x}=1$,

$$
K_{i}(x, \vartheta)=0 \Rightarrow \partial K_{i}(x, \vartheta)\left[\begin{array}{c}
f(x) \\
\partial f(x) \vartheta
\end{array}\right]>0
$$

Proof: (i) For $K(x, \vartheta) \geq 0$, consider $K_{i}(x, \vartheta)=0$. Then, $K_{i}(x, \rho \vartheta)=0$ for any $\rho>0$, thus $\left[\partial_{\vartheta} K_{i}(x, \vartheta)\right] \vartheta=$ 0 . Since $\mathcal{C}$ is a compact set, by continuity, there exists $k_{1}>0$ (sufficiently large) and $\varepsilon>0$ (sufficiently small) such that

$$
0 \leq K_{i}(x, \vartheta) \leq \varepsilon \Rightarrow\left[\partial_{\vartheta} K_{i}(x, \vartheta)\right] \vartheta \leq k_{1} \varepsilon .
$$

(ii) Exploiting the compactness of $\mathcal{C}$ again, (17) guarantees that there exist $k_{2}>0$ and a small $\varepsilon>0$ such that $K_{i}(x, \vartheta)=0 \Rightarrow \partial K_{i}(x, \vartheta)\left[f(x)^{T} \partial f(x)^{T} \vartheta\right]^{T} \geq$ $k_{2}+\bar{\lambda} k_{1} \varepsilon$ where $\bar{\lambda}:=\max _{x \in \mathcal{C},|\vartheta|_{x}=1, \mathcal{K}(x, \vartheta) \geq 0} \lambda(x, \vartheta)$ and $\lambda(x, \vartheta)$ refers to $(15)$. By continuity, for $\varepsilon$ sufficiently small there exists $k_{3}>0$ such that

$$
\begin{aligned}
& 0 \leq K_{i}(x, \vartheta) \leq \varepsilon \Rightarrow \\
& \partial K_{i}(x, \vartheta)\left[\begin{array}{c}
f(x) \\
\partial f(x) \vartheta
\end{array}\right] \geq k_{2}+\bar{\lambda} k_{1} \varepsilon-k_{3} \varepsilon \geq \frac{k_{2}}{2}+\bar{\lambda} k_{1} .
\end{aligned}
$$

for any $x \in \mathcal{C}$ and $|\vartheta|_{x}=1$ such that $\mathcal{K}(x, \vartheta) \geq 0$.

(iii) We follow now the proof of Theorem 2, combining (i) and (ii). Consider any trajectory $\left(\psi(\cdot, x), \partial_{x} \psi(\cdot, x) \delta x\right) \in$ $\delta \Sigma$, define $\vartheta(t):=\frac{\partial_{x} \psi(t, x) \delta x}{\left|\partial_{x} \psi(t, x) \delta x\right|_{\psi(t, x)}}$ and $x(t):=\psi(t, x)$. Take $T:=\frac{2 \varepsilon}{k_{2}}$ and suppose that for all $t \in[0, T], 0 \leq$ $K_{i}(x(t), \vartheta(t))<\varepsilon$. Combining (15), (18) and (19), we get

$$
\begin{aligned}
& \frac{d}{d t} K_{i}(x(t), \vartheta(t)) \geq \\
& \geq \frac{\varepsilon}{T}+\bar{\lambda} k_{1} \varepsilon-\underbrace{\lambda(x(t), \vartheta(t))}_{\leq \bar{\lambda}} \underbrace{\left[\partial_{\vartheta(t)} K_{i}(x(t), \vartheta(t))\right] \vartheta(t)}_{\leq k_{1} \varepsilon} \geq \frac{\varepsilon}{T}
\end{aligned}
$$


which leads to a contradiction by integration over the interval $[0, T]$, as in the proof of Theorem 2 .

Theorem 3 is illustrated in the following examples.

Example 3: Cooperative systems. A nonlinear system $\Sigma$ given by $\dot{x}=f(x), x \in \mathbb{R}^{n}$, is cooperative if its Jacobian $\partial f(x)$ has nonnegative off-diagonal elements, [18]. Cooperative systems are differentially positive systems in $\mathcal{X}:=\mathbb{R}^{n}$ with respect to the constant polyhedral cone field given by the positive orthant, that is, $\mathcal{K}(x):=\mathbb{R}_{+}^{n}$ for any $x \in \mathcal{X}$ (Theorem 1). Given any compact and forward invariant region $\mathcal{C} \subseteq \mathbb{R}^{n}, \Sigma$ is strictly differentially positive if the off-diagonal elements of $\partial f(x)$ are strictly positive (Theorem 3). To see this, consider the standard inner product in $\mathbb{R}^{n}$ and the cone field (4) given by $F_{i}(x):=e_{i}$ for each $x \in \mathcal{C}$ and $i \in \mathcal{I}:=\{1, \ldots, n\}$, where $e_{i}$ is the canonical base. Then, $\delta x \in \mathcal{K}(x)$ reads $e_{i}^{T} \delta x \geq 0$ for all $i$. (17) reads $e_{i}^{T} \delta x=0 \Rightarrow e_{i}^{T} \partial f(x) \delta x>0$ for $|\delta x|_{2}=1$, which is equivalent to $e_{i}^{T} \partial f(x) e_{j}>0$ for all $i, j \in \mathcal{I}, i \neq j$. $\lrcorner$

Example 4: Differential positivity of the pendulum. Consider the nonlinear pendulum given by the equations $\dot{\vartheta}=v$, $\dot{v}=-\sin (\vartheta)-k v+u$. The linearization reads $\dot{\delta \vartheta}=\delta v$, $\dot{\delta v}=-\cos (\vartheta) \delta \vartheta-k \delta v$. Theorem 3 guarantees that for any $k>2$, any input $u$, and any compact and forward invariant region $\mathcal{C}$, the pendulum is strictly differentially positive in $\mathcal{C}$ with respect to the cone field (4) given by

$$
\delta \vartheta \geq 0 \quad \delta \vartheta+\delta v \geq 0
$$

For instance, for $\left|\left[\begin{array}{ll}\delta \vartheta & \delta v\end{array}\right]^{T}\right|=1$, (i) $\delta \vartheta=0, \delta \vartheta+\delta v>0$. Then $\dot{\delta} \vartheta=\delta v>-\delta \vartheta>0$. (ii) $\delta \vartheta>0, \delta \vartheta+\delta v=0$. Then, $\dot{\delta \vartheta}+\dot{\delta v}=\delta v-\cos (\vartheta) \delta \vartheta-k \delta v \geq(k-1-1) \delta \vartheta>0$.

Revisiting [7, Section VIII], Theorem 6 below can be used to establish the existence of limit cycles for $u>1$.

\section{ASYMPTOTIC BEHAVIOR}

\section{A. Stable attractors}

It is well known that under mild conditions almost every bounded trajectory of a monotone system converges to a fixed point [8], [18]. This fundamental result has been recently revisited in [7, Corollary 5], with a new proof based on differential positivity. The next theorem extends this result to a larger class of systems.

Given a compact set $\mathcal{C} \subseteq \mathcal{X}$ we say that a conal curve $\gamma: \mathbb{R} \rightarrow \mathcal{X}$ intersects the boundary of $\mathcal{C}$ twice if for any $s \in \mathbb{R}$ such that $\gamma(s) \in \mathcal{C}$, there exists an interval $\underline{s} \leq s \leq \bar{s}$ such that $\gamma(\underline{s}), \gamma(\bar{s}) \notin \mathcal{C}$.

Theorem 4: Under Assumption 1, consider a strictly differentially positive system $\Sigma$ with respect to the cone field $\mathcal{K}(x)$ in a compact forward invariant region $\mathcal{C} \subseteq \mathcal{X}$. Suppose that every conal curve $\gamma: \mathbb{R} \rightarrow \mathcal{X}$ intersects the boundary of $\mathcal{C}$ twice. Then, from almost every initial condition in $\mathcal{C}$ the trajectories of $\Sigma$ converge asymptotically to a fixed point. $\lrcorner$

The next theorem exploits the combination of differential positivity with the existence of an invariant vector field $v(x) \in \mathcal{K}_{\varepsilon}(x)$. It shows that the trajectories of the system converge asymptotically to a one dimensional attractor given by the image of an integral curve of $v(x)$.
Theorem 5: Under Assumption 1, consider a strictly differentially positive system $\Sigma$ with respect to the cone field $\mathcal{K}(x)$ in a compact forward invariant region $\mathcal{C} \subseteq \mathcal{X}$. Let $\varepsilon>0$ and suppose that there exists a complete vector field $v(x) \in \mathcal{K}_{\varepsilon}(x) \backslash\{0\}$ such that

$$
\begin{gathered}
\limsup _{t \rightarrow \infty}\left|\partial \psi_{t}(x) v(x)\right|_{\psi_{t}(x)}<\infty \\
v\left(\psi_{t}(x)\right)=\frac{\partial \psi_{t}(x) v(x)}{\left|\partial \psi_{t}(x) v(x)\right|_{\psi_{t}(x)}} \quad \forall x \in \mathcal{C}, \forall t \geq 0 .
\end{gathered}
$$

Then, there exists an integral curve of $v(x)$ whose image is an attractor for all the trajectories of $\Sigma$ from $\mathcal{C}$.

Finding the vector field that satisfies (22) and (23) can be difficult in general. However, the presence of symmetries in the system makes (22) and (23) tractable conditions. An example is given by consensus dynamics [13], [16] where $n$ agents communicate with their neighborhoods to achieve consensus, typically given by the manifold $x_{1}=\cdots=$ $x_{n}$. The invariance of the consensus manifold dictates the symmetry $\partial f(x) \mathbf{1}=0, \mathbf{1}:=\left[\begin{array}{lll}1 & \ldots & 1\end{array}\right]^{T} \in T_{x} \mathcal{X}$, which makes (22) and (23) trivially verified by $v(x):=\frac{1}{\sqrt{n}}$. This observation is used in Section VI for the analysis of the Kuramoto model.

Replacing $v(x)$ in Theorem 5 with the system vector field $f(x)$, the next theorem gives conditions for the existence of attractive limit cycles. This result is compatible with Theorem 5, since conal curves can be closed curves.

Theorem 6: Under Assumption 1, consider a strictly differentially positive system $\Sigma$ with respect to the cone field $\mathcal{K}(x)$ in a compact forward invariant region $\mathcal{C} \subseteq \mathcal{X}$. Suppose that $\mathcal{C}$ does not contain any fixed point. If $f(x) \in \mathcal{K}_{\varepsilon}(x) \backslash\{0\}$ for any $x \in \mathcal{C}$, for some $\varepsilon>0$, then there exists a unique attractive periodic orbit contained in $\mathcal{C}$.

Remark 1: Converse results for hyperbolic limit cycles can be found in [11]. Theorem 6 revisits [7, Corollary 2], which requires differential positivity in the whole manifold $\mathcal{X}$, a condition weakened by Theorem 6 . The key step for this result is a new proof that does not use the so-called Perron-Frobenius vector field of [7, Section VI].

\section{B. Proofs}

Proof of Theorem 4. Suppose that for some $x \in \mathcal{X}$, the trajectory $\psi(\cdot, x)$ does not converge to a fixed point and denote by $\omega(x)$ the $\omega$-limit set of $x$. Then, there exists a sequence of time instant $t_{k} \rightarrow \infty$ as $k \rightarrow \infty$ and $c>0$ such that $\left|f\left(\psi\left(t_{k}, x\right)\right)\right|>c$, for all $k \in \mathbb{N}$. By continuity, since $\psi(t, x) \in \mathcal{C}$ for all $t \geq 0$, there exists a small constant $\rho>0$ such that $|f(\psi(t, x))|>c$ for all $t \in\left[t_{k}-\rho, t_{k}+\rho\right]$.

Recall also that $(\psi(\cdot, x), f(\psi(\cdot, x)) \in \delta \Sigma$ since $\frac{d}{d t} f(\psi(t, x))=\partial f(\psi(t, x)) f(\psi(t, x))$. Thus, either (i) there exists $\tau \geq 0$ such that $f(\psi(t, x)) \in-\mathcal{K}(\psi(t, x)) \cup$ $\mathcal{K}(\psi(t, x))$ for all $t \geq \tau$, or (ii) $f(\psi(t, x)) \notin-\mathcal{K}(\psi(t, x)) \cup$ $\mathcal{K}(\psi(t, x))$ for all $t \geq 0$.

For (i), note that the points $\psi_{t+\tau}(x)$ for $t \geq 0$ belong to the image of a conal curve, since $\frac{d}{d t} \psi_{t+\tau}(x)=f\left(\psi_{t+\tau}(x)\right) \in$ $\mathcal{K}\left(\psi_{t+\tau}(x)\right)$ (or $\left.-f\left(\psi_{t+\tau}(x)\right) \in \mathcal{K}\left(\psi_{t+\tau}(x)\right)\right)$ for any $t \geq 0$. Then, since $\left|f\left(\psi_{t+\tau}(x)\right)\right| \geq c$ for all $t+\tau \in\left[t_{k}-\rho, t_{k}+\right.$ 
$\rho]$, exploiting the fact that every conal curve intersects the boundary of $\mathcal{C}$ twice, there exists a time $T>0$ such that $\psi_{T+\tau}(x) \notin \mathcal{C}$. This contradicts the forward invariance of $\mathcal{C}$.

For (ii), we show that the basin of attraction of $\mathcal{A}:=\omega(x)$ has dimension $n-1$ at most. We need a preliminary result. Consider any $\delta x \in \mathcal{K}_{\varepsilon}(x)$ for some $\varepsilon>0$. Then, for $\alpha>0$ sufficiently large $f(x)+\alpha \delta x \in \mathcal{K}(x)$. Thus, by projective contraction, $\lim _{t \rightarrow \infty} d_{\mathcal{K}\left(\psi_{t}(x)\right)}\left(\partial \psi_{t}(x)[f(x)+\alpha \delta x], \partial \psi_{t}(x) \alpha \delta x\right)=$ $\lim _{t \rightarrow \infty} d_{\mathcal{K}\left(\psi_{t}(x)\right)}\left(\alpha \partial \psi_{t}(x) \delta x+f\left(\psi_{t}(x)\right), \alpha \partial \psi_{t}(x) \delta x\right)=0$. Since $\left|f\left(\psi_{t_{k}}(x)\right)\right| \geq c$, it follows that

$$
\lim _{t \rightarrow \infty}\left|\partial \psi_{t}(x) \delta x\right|_{\psi_{t}(x)}=\infty \quad \forall \delta x \in \operatorname{int} \mathcal{K}(x)
$$

By contradiction, suppose now that the basin of attraction $\mathcal{B}_{\mathcal{A}}$ has dimension $n$. Consider a conal curve $\gamma$ such that $\gamma(0) \in \mathcal{A}$ and $\gamma(s) \in \mathcal{B}_{\mathcal{A}} \backslash\{A\}$ for all $s \in[0, \bar{s}] \subseteq$ $\mathbb{R}$. By assumption, for all $s \in[0, \bar{s}], \psi_{t}(\gamma(s))$ converges asymptotically to $\mathcal{A}$ as $t \rightarrow \infty$. Moreover, $f\left(\psi_{t}(\gamma(s))\right) \notin$ $\mathcal{K}\left(\psi_{t}(\gamma(s))\right)$ for all $t \geq 0$, and $\left|f\left(\psi_{t_{k}}(\gamma(s))\right)\right| \geq c$ for $k$ sufficiently large. From (24), it follows that

$$
\lim _{t \rightarrow \infty}\left|\partial \psi_{t}(x) \dot{\gamma}(s)\right|_{\psi_{t}(\gamma(s))}=\infty
$$

From (25), the length of the curve $\psi_{t}(\gamma(\cdot))$ grows unbounded as $t \rightarrow \infty$, since $\ell\left(\psi_{t}(\gamma(\cdot))\right)=\int_{0}^{1}\left|\frac{d}{d s} \psi_{t}(\gamma(s)) d s\right|_{\psi_{t}(\gamma(s))}=$ $\int_{0}^{1}\left|\partial \psi_{t}(\gamma(s)) \dot{\gamma}(s)\right|_{\psi_{t}(\gamma(s))} d s$. It follows that there exists $T>0$ such that $\psi_{T}(\gamma(\bar{s})) \notin \mathcal{C}$, contradicting the forward invariance of the set.

Proof of Theorem 5. [(i) boundedness] Under the assumptions of the theorem, we prove that for any given $\delta x \in T_{x} \mathcal{X}$,

$$
\lim _{t \rightarrow \infty}\left|\partial \psi_{t}(x) \delta x\right|_{\psi_{t}(x)}<\infty .
$$

Consider first the case $\partial \psi_{t}(x) \delta x \notin-\mathcal{K}\left(\psi_{t}(x)\right) \cup$ $\mathcal{K}\left(\psi_{t}(x)\right)$ for all $t \geq 0$. Then for $\alpha>0$ sufficiently large $\delta x+\alpha v(x) \in \mathcal{K}(x)$, thus Lemma 1 gives $\lim _{t \rightarrow \infty} d_{\mathcal{K}\left(\psi_{t}(x)\right)}\left(\partial \psi_{t}(x)[\alpha v(x)+\delta x], \partial \psi_{t}(x) v(x)\right)=$ $\lim _{t \rightarrow \infty} d_{\mathcal{K}\left(\psi_{t}(x)\right)}^{t \rightarrow \infty}\left(\alpha \partial \psi_{t}(x) v(x)+\partial \psi_{t}(x) \delta x, \alpha \partial \psi_{t}(x) v(x)\right)=0$. Since $\left|\partial \psi_{t}(x) v(x)\right|_{\psi_{t}(x)}$ is bounded, it follows that

$$
\lim _{t \rightarrow \infty}\left|\partial \psi_{t}(x) \delta x\right|_{\psi_{t}(x)}=0 \text {. }
$$

Consider now the case $\partial \psi_{T}(x) \delta x \in \mathcal{K}\left(\psi_{T}(x)\right)$ for some $T \geq 0$. If $\partial \psi_{T}(x) \delta x \in-\mathcal{K}\left(\psi_{T}(x)\right)$, consider the symmetric trajectory $\partial \psi_{T}(x)[-\delta x] \in \mathcal{K}\left(\psi_{T}(x)\right)$. Suppose that $\lim _{t \rightarrow \infty}\left|\partial \psi_{t}(x) \delta x\right|_{\psi_{t}(x)}=\infty$. Then, by projective contraction $d_{\mathcal{K}\left(\psi_{t+T}(x)\right)}\left(\partial \psi_{t+T}(x) \delta x, \partial \psi_{t+T}(x) v(x)\right)=0$. Therefore, for $t$ sufficiently large, $\partial \psi_{t+T}(x) v(x)$ is almost parallel to $\partial \psi_{t+T}(x) \delta x$. By linearity of $\partial \psi_{t+T}(x)$, it follows that $\partial \psi_{t+T}(x) v(x)$ grows unbounded, contradicting (22).

[(ii) horizontal contraction] We show that any pair of points in $\mathcal{C}$ converge to the image of an integral curve of $v(x)$. Consider any curve $\gamma_{0}(\cdot):[0,1] \rightarrow \mathcal{C}$ connecting two different points $\gamma_{0}(0), \gamma_{0}(1) \in \mathcal{C}$. The evolution of the curve along the flow is given by $\gamma_{t}(s):=\psi_{t}(\gamma(s))$. Note that $\frac{d}{d s} \gamma_{t}(s)=\partial \psi_{t}(\gamma(s)) \dot{\gamma}(s)$. From (i), either $\lim _{t \rightarrow \infty} \frac{d}{d s} \gamma_{t}(s)=0$ by (27), or there exists $T$ such that $\frac{d}{d s} \gamma_{t}(s) \in-\mathcal{K}\left(\gamma_{t}(s)\right) \cup$
$\mathcal{K}\left(\gamma_{t}(s)\right)$ for all $t \geq T$. In this last case, using (26), $\frac{d}{d s} \gamma_{t}(s)$ is bounded, and $d_{\mathcal{K}\left(\gamma_{t}(s)\right)}\left(\frac{d}{d s} \gamma_{t}(s), \partial \psi_{t}(\gamma(s)) v(\gamma(s))\right)=0$. Thus, in the limit of $t \rightarrow \infty, \frac{d}{d s} \gamma_{t}(s)$ becomes parallel to $v\left(\gamma_{t}(s)\right)$. It follows that $\psi_{t}(\gamma(s))$ belongs to the image of an integral curve of $v(x)$.

[(iii) uniqueness] By contradiction, let $\mathcal{A}_{1}$ and $\mathcal{A}_{2}$ be images of two distinct attractive integral curves of $v(x)$. Take $x_{1} \in \mathcal{A}_{1}, x_{2} \in \mathcal{A}_{2}$, and consider a new curve $\gamma$ connecting them. Along the flow, $\psi_{t}(\gamma(s))$ converges to an integral curve of $v(x)$. Thus, by completeness, $\mathcal{A}_{1}$ and $\mathcal{A}_{2}$ are subsets of the image of the same integral curve of $v(x)$, contradicting the initial hypotesis.

Proof of Theorem 6. From the conditions of the theorem, there exists $0<c_{1}<c_{2}$ such that $c_{1} \leq|f(x)| \leq c_{2}$ for all $x \in \mathcal{C}$. Reasoning like in the proof of Theorem 5 by replacing $v(x)$ by $f(x)$, we get

$$
\lim _{t \rightarrow \infty}\left|\partial \psi_{t}(x) \delta x\right|_{\psi_{t}(x)}<\infty
$$

for any $x \in \mathcal{C}$ and $\delta x \in T_{x} \mathcal{X}$. Furthermore, we can conclude that there exists a unique integral curve of the vector field $f(x)$ - a trajectory - whose image is an attractor for every trajectory of $\Sigma$ from $\mathcal{C}$. In what follows we show that this curve is closed, by following the proof of [7, Corollary 2].

Let $\psi(\cdot, x)$ be such a curve, for some $x \in \mathcal{C} . \psi(\cdot, x)$ does not converge to a fixed point and belongs to a compact set, therefore there is a point $x^{*}=\psi\left(t^{*}, x\right)$ whose neighborhood $\mathcal{B}_{\rho}\left(x^{*}\right)$ is visited by the trajectory infinitely many times, for any $\rho>0$. Take $\rho>0$ sufficiently small and let $\mathcal{S} \subseteq \mathcal{C}$ be a transversal manifold to the trajectories of $\Sigma$ in $\mathcal{B}_{\rho}\left(x^{*}\right)$, namely $f(x) \notin T_{x} \mathcal{S}$ for all $x \in \mathcal{S} \cap \mathcal{B}_{\rho}\left(x^{*}\right)$ and $x^{*} \in \mathcal{S}$. Consider a sequence of time instants $t_{k} \rightarrow \infty$ for $k \rightarrow \infty$ such that $\psi\left(t_{k}, x^{*}\right) \in \mathcal{S} \cap \mathcal{B}_{\rho}\left(x^{*}\right)$, and the subsequence of time instants $t_{k_{j}} \rightarrow \infty$ for $j \rightarrow \infty$ such that $\psi\left(t_{k_{j}}, x^{*}\right) \in$ $\mathcal{S} \cap \mathcal{B}_{\frac{\rho}{3}}\left(x^{*}\right)$,

Consider now any curve $\gamma(\cdot):[0,1] \rightarrow \mathcal{S} \cap \mathcal{B}_{\rho}\left(x^{*}\right)$ such that the length of $\ell(\gamma(\cdot))=\int_{0}^{1}|\dot{\gamma}(s)|_{\gamma(s)} d s$ is less than or equal to $\rho \in \mathbb{R}$ and $\gamma(0)=x^{*}$. By (28), there exists $c_{3} \geq 1$ such that $\limsup _{t \rightarrow \infty} \ell\left(\psi_{t}(\gamma(\cdot))\right) \leq c_{3} \rho$. Furthermore, $\lim _{t \rightarrow \infty} d_{\mathcal{K}\left(\psi_{t}(x)\right)}\left(\frac{d}{d s} \psi_{t}(\gamma(s)), f\left(\psi_{t}(\gamma(s))\right)=\right.$ 0 for all $s \in[0,1]$, that is, in the limit of $t \rightarrow \infty$, the image of $\psi_{t}(\gamma(\cdot))$ converges asymptotically to the image of the attractive integral curve of the vector field $f(x)$. Thus, because of the transversality of $\mathcal{S}$, the combination of the bound $\lim \sup _{t \rightarrow \infty} \ell\left(\psi_{t}(\gamma(\cdot))\right) \leq c_{3} \rho$ and of the convergence of the Hilbert metric guarantees that there exist $k$ sufficiently large and $\tau_{k}(s)$ typically small, such that $\psi\left(t_{k}+\tau_{k}(s), \gamma(s)\right) \in \mathcal{S} \cap \mathcal{B}_{\frac{\rho}{3}}\left(\psi\left(t_{k}, x^{*}\right)\right), \forall s \in[0,1]$. Thus, there exists a $j$ sufficiently large such that $\psi\left(t_{k_{j}}+\right.$ $\left.\tau_{k_{j}}(s), \gamma(s)\right) \in \mathcal{S} \cap \mathcal{B}_{\frac{\rho}{3}}\left(\psi\left(t_{k_{j}}, x^{*}\right)\right) \subset \mathcal{S} \cap \mathcal{B}_{\frac{2 \rho}{3}}\left(x^{*}\right)$. It follows that the return map on $\mathcal{S}$ is necessarily a contraction, which implies that the attractor is a closed curve.

\section{EXTENDED EXAMPLE: ALL-TO-ALL KURAMOTO}

We study the synchronization of Kuramoto dynamics for the case of all-to-all coupling with homogeneous velocities at zero using the results of Section V. Differential positivity is verified by using the geometric conditions of Section IV. 
Consider the interconnection of $n$ agents (phase variables) given by $\dot{\vartheta}_{k}=\frac{1}{n} \sum_{i=1}^{n} \sin \left(\vartheta_{i}-\vartheta_{k}\right)$ where $\vartheta_{k} \in \mathbb{S}$. The synchronization manifold is given by $\mathcal{A}:=\left\{\vartheta \in \mathbb{S}^{n} \mid \vartheta_{1}=\right.$ $\left.\cdots=\vartheta_{n}\right\}$, where $\vartheta:=\left[\begin{array}{lll}\vartheta_{1} & \ldots & \vartheta_{n}\end{array}\right]^{T}, k \in \mathcal{I}:=\{1, \ldots, n\}$. Using $1:=\left[\begin{array}{lll}1 & \ldots & 1\end{array}\right]^{T}$, the prolonged dynamics reads

$$
\left\{\begin{aligned}
\dot{\vartheta} & =\frac{1}{n} \mathbf{S}(\vartheta) \mathbf{1} \\
\dot{\delta \vartheta} & =\frac{1}{n} \mathbf{C}(\vartheta) \delta \vartheta
\end{aligned} \quad(\vartheta, \delta \vartheta) \in T \mathbb{S}^{n}\right.
$$

where for $k, i \in \mathcal{I}, \mathbf{S}_{k i}(\vartheta):=\sin \left(\vartheta_{i}-\vartheta_{k}\right), \mathbf{C}_{k k}(\vartheta):=$ $-\sum_{i \neq k} \cos \left(\vartheta_{i}-\vartheta_{k}\right)$, and $\mathbf{C}_{k i}(\vartheta):=\cos \left(\vartheta_{i}-\vartheta_{k}\right)$ for $k \neq j$.

The invariance of the synchronization manifold is captured by the identity $\mathbf{C}(\vartheta) \mathbf{1}=0$. The constant vector field $v(\vartheta)=$ $\frac{1}{\sqrt{n}}$ satisfies the conditions of Theorem 5. Thus, exploiting the theorem, given a compact and forward invariant region $\mathcal{C}$, all trajectories from $\mathcal{C}$ converge asymptotically to the synchronization manifold $\mathcal{A} \subseteq \mathcal{C}$, provided that the system is strictly differentially positive with respect to some cone field $\mathcal{K}(\vartheta)$ such that $\mathbf{1} \in \operatorname{int} \mathcal{K}(\vartheta)$ (interior of $\mathcal{K}(\vartheta)$ ).

Define $\Pi:=\left[I-\frac{\mathbf{1 1}^{T}}{n}\right]$ and consider the centroid $\rho e^{i \phi}:=$ $\frac{1}{n} \sum_{k \in \mathcal{I}} e^{i \vartheta_{k}} . \rho \in[0,1]$ is a measure of the synchrony of the phase variables [17, Section III]. We show that the system is strictly differentially positive in any compact and forward invariant region $\mathcal{C} \subset \mathbb{S}^{n}$ with respect to the quadratic cone field (5) given by

$$
\begin{aligned}
& K_{1}(\vartheta, \delta \vartheta):=\mathbf{1}^{T} \delta \vartheta \\
& K_{2}(\vartheta, \delta \vartheta):=e^{2 \lambda \rho} \delta \vartheta^{T} \mathbf{1 1}^{T} \delta \vartheta-\delta \vartheta^{T} \Pi \delta \vartheta
\end{aligned}
$$

where $\lambda>0$ is a parameter defined below. An illustration is provided in Figure 3.

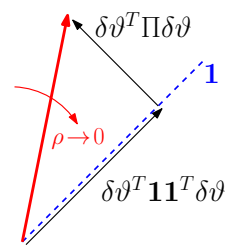

Fig. 3. Increasing $\rho$ widens the cone. The cone is widest at $\rho=1$.

For $K_{2}(\vartheta, \delta \vartheta)=0$, using the identities $\mathbf{1}^{T} \mathbf{C}(\vartheta)=0$ and $\Pi \mathbf{C}(\vartheta)=\mathbf{C}(\vartheta), \frac{d}{d t} K_{2}(\vartheta, \delta \vartheta)$ along the flow of the prolonged dynamics reads

$$
2 \lambda \dot{\rho} e^{2 \lambda \rho} \delta \vartheta^{T} \mathbf{1 1}^{T} \delta \vartheta-\delta \vartheta^{T}\left(\mathbf{C}(\vartheta)^{T}+\mathbf{C}(\vartheta)\right) \delta \vartheta
$$

where, following [17, Section III] and [6, Section VII], $\dot{\rho}=\frac{\rho}{n} \sum_{k \in \mathcal{I}} \sin \left(\vartheta_{k}-\phi\right)^{2}$. In particular, $\dot{\rho}=0$ for $\rho=0$ (balanced phases, max spread on the circle) or for $\sum_{k \in \mathcal{I}} \sin \left(\vartheta_{k}-\phi\right)^{2}=0$, which occurs on isolated critical points given by $n-m$ phases synchronized at $\phi+2 j \pi$ and $m$ phases synchronized at $\phi+\pi+2 j \pi$, for $j \in \mathbb{N}$ and $0 \leq m \leq \frac{n}{2}$ (for an extended analysis see [17, Section III]).

For $\vartheta \in \mathbb{S}_{\pi / 2}^{n}:=\left\{\vartheta \in \mathbb{S}^{n}|| \vartheta_{k}-\vartheta_{j} \mid<\frac{\pi}{2}, \forall k, j \in \mathcal{I}\right\}$, the quantity $-\delta \vartheta^{T}\left(\mathbf{C}(\vartheta)^{T}+\mathbf{C}(\vartheta)\right) \delta \vartheta>0$. For $\vartheta \notin \mathbb{S}_{\pi / 2}^{n}$, we can design $\lambda$ so that the first term $2 \lambda \dot{\rho} e^{2 \lambda \rho} \delta \vartheta^{T} \mathbf{1 1}^{T} \delta \vartheta$ in (31) dominates $-\delta \vartheta^{T}\left(\mathbf{C}(\vartheta)^{T}+\mathbf{C}(\vartheta)\right) \delta \vartheta$. Given any compact and forward invariant set $\mathcal{C} \subset \mathbb{S}^{n}$ that does not contain any balanced phase $(\rho=0)$ or saddle point $\left(\sum_{k \in \mathcal{I}} \sin \left(\vartheta_{k}-\right.\right.$ $\phi)^{2}=0$ ), there exists a sufficiently small $\varepsilon>0$ such that $\sum_{k \in \mathcal{I}} \sin \left(\vartheta_{k}-\phi\right)^{2}>\varepsilon$ and $\rho>0$ for every $\vartheta \in \mathcal{C}$. Thus, there exists a sufficiently large $\lambda$ such that $K_{2}(\vartheta, \delta \vartheta)=0 \Rightarrow$ $\frac{d}{d t} K_{2}(\vartheta, \delta \vartheta)>0$.

\section{CONCLUSION}

Differential positivity provides a number of methods for the analysis of simple attractors of nonlinear (closed) systems, capturing bistable and periodic behaviors. The introduction of compact sets simplifies the use of differential positivity in applications. The theory have been illustrated on several examples based on polyhedral and quadratic cone fields. Future research directions will study differential positivity on open systems.

\section{REFERENCES}

[1] D. Angeli and E.D. Sontag. Monotone control systems. IEEE Transactions on Automatic Control, 48(10):1684 - 1698, 2003.

[2] G. Birkhoff. Extensions of Jentzsch's theorem. Transactions of the American Mathematical Society, 85(1):pp. 219-227, 1957.

[3] P.J. Bushell. Hilbert's metric and positive contraction mappings in a Banach space. Archive for Rational Mechanics and Analysis, 52(4):330-338, 1973.

[4] P.E. Crouch and A.J. van der Schaft. Variational and Hamiltonian control systems. Lecture notes in control and information sciences. Springer, 1987.

[5] L. Farina and S. Rinaldi. Positive linear systems: theory and applications. Pure and applied mathematics (John Wiley \& Sons). Wiley, 2000.

[6] F. Forni and R. Sepulchre. A differential Lyapunov framework for contraction analysis. IEEE Transactions on Automatic Control, 59(3):614-628, 2014

[7] F. Forni and R. Sepulchre. Differentially positive systems. Accepted in IEEE Transactions on Automatic Control (Available at http://arxiv.org/abs/1405.6298), 2014.

[8] M.W. Hirsch. Stability and convergence in strongly monotone dynamical systems. Journal für die reine und angewandte Mathematik, 383:1-53, 1988.

[9] M.W. Hirsch. Fixed points of monotone maps. Journal of Differential Equations, 123(1):171 - 179, 1995.

[10] B. Lemmens and R.D. Nussbaum. Nonlinear Perron-Frobenius theory. Cambridge tracts in mathematics, 189. Cambridge University Press, 2012.

[11] A. Mauroy, F. Forni, and R. Sepulchre. An operator-theoretic approach to differential positivity. In Submitted to the 54th IEEE Conference on Decision and Control, 2015.

[12] L. Moreau. Stability of continuous-time distributed consensus algorithms. In 43rd IEEE Conference on Decision and Control, volume 4, pages 3998 - 4003, 2004.

[13] R. Olfati-Saber, J.A. Fax, and R.M. Murray. Consensus and cooperation in networked multi-agent systems. Proceedings of the IEEE, 95(1):215-233, Jan 2007.

[14] A. Rantzer. Distributed control of positive systems. ArXiv e-prints, 2012.

[15] B. Roszak and E.J. Davison. Necessary and sufficient conditions for stabilizability of positive LTI systems. Systems \& Control Letters, 58(7):474 - 481, 2009

[16] R. Sepulchre. Consensus on nonlinear spaces. In 8th IFAC Symposium on Nonlinear Control Systems, 2010.

[17] R. Sepulchre, D.A. Paley, and N.E. Leonard. Stabilization of planar collective motion: All-to-all communication. IEEE Transactions on Automatic Control, 52(5):811-824, 2007.

[18] H.L. Smith. Monotone Dynamical Systems: An Introduction to the Theory of Competitive and Cooperative Systems, volume 41 of Mathematical Surveys and Monographs. American Mathematical Society, 1995.

[19] C.B. Zhai and Z.D. Liang. Hilbert's projective metric and the norm on a Banach space. Journal of Mathematical Research with Applications, 31(1):91-99, 2011. 